\title{
Endoscopic ultrasound-guided biliary rendezvous with primary sphincteroplasty for stone extraction from an "inverse pitcher" papilla
}

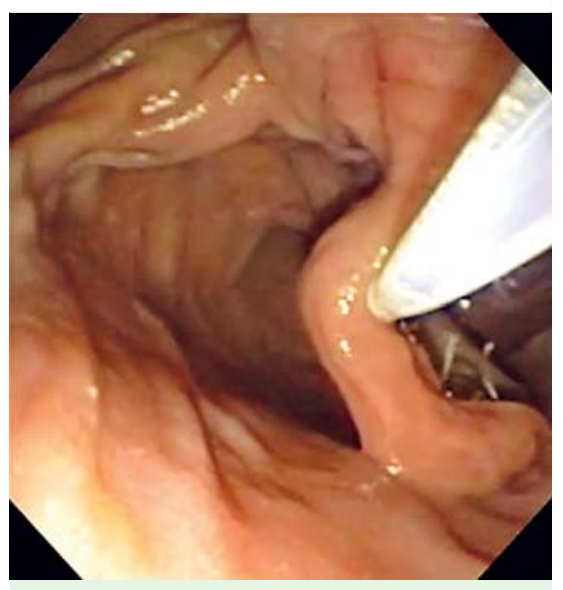

Fig. 1 Endoscopic view of the inverse-pitcher ampulla in a 55-year-old Caucasian woman presenting with ductal calculus.

A 55-year-old Caucasian woman with common bile ductal calculus was referred for endoscopic retrograde cholangiopancreatography (ERCP) after a failed procedure at another facility. Endoscopy revealed a hooded, elongated, inverse pitcher-type diverticulum with an invisible ampulla. The "trunk" of this diverticulum was at least $5 \mathrm{~cm}$ long ( Fig. 1). Multiple attempts by two experienced endoscopists to identify and cannulate the ampulla were futile. Therefore, a linear array echoendocope was advanced into the duodenum, and a 9-mm bile duct with 8 -mm calculus was identified. The duct was punctured with a 19-G flexible needle (Boston Scientific, Natick, Massachusetts, USA). After contrast injection, a 0.025-inch guidewire (Visiglide, Olympus, Melville, New York, USA) was placed in the bile duct and slowly advanced through the ampulla ( Fig.2) [1]. Following withdrawal of the echoendoscope, the guidewire was left in situ and a duodenoscope was advanced. The duodenal portion of the guidewire was removed through the biopsy channel of the duodenoscope while the biliary end of the guidewire was advanced slowly by an assistant. A biliary brush catheter (Cook Medical, Bloomington, Indiana, USA) was passed over the guidewire after removal of the metal brush. Contrast injection confirmed placement of the catheter tip inferior to the bile duct puncture site. Another 0.025-inch guidewire (Visiglide) was advanced through the other channel of the catheter and placed into the intrahepatic bile duct followed by removal of the rendezvous guidewire. A biliary sphincterotomy could not be carried out as the ampulla was not visible. Therefore, a primary balloon sphincteroplasty with an $8-\mathrm{mm}$ balloon

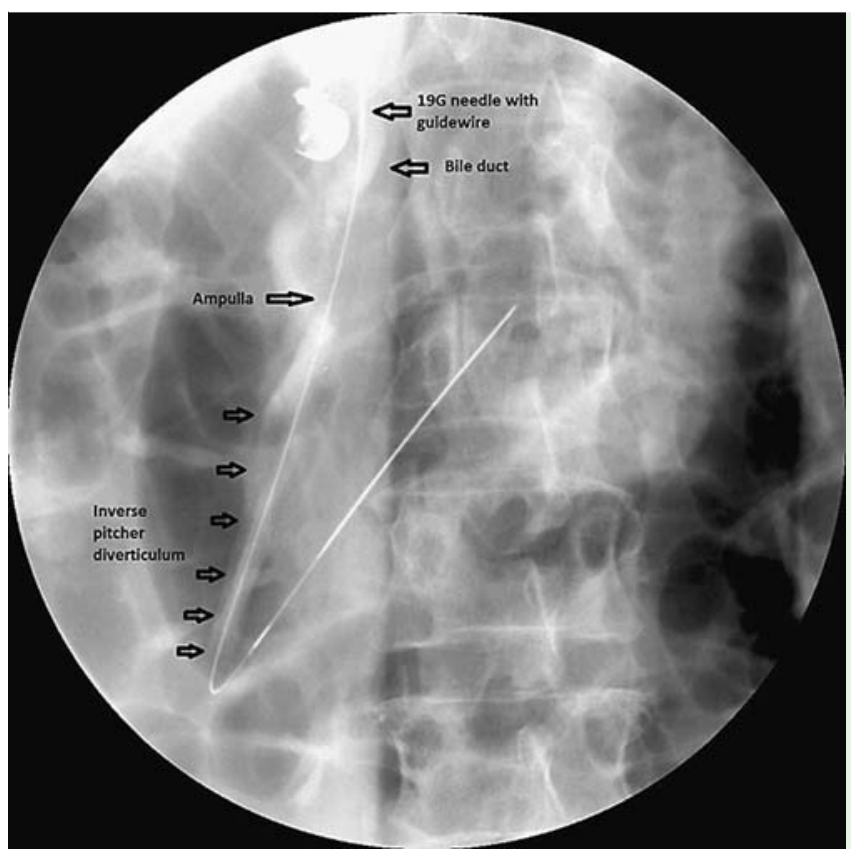

Fig. 2 Fluoroscopic image of endoscopic ultrasound (EUS)-guided bile duct puncture and placement of guidewire into the duodenum. Note the elongated duodenal diverticulum. enabled removal of the calculus with an extraction balloon. A $10-\mathrm{Fr}, 15-\mathrm{cm}$ plastic biliary stent was then placed to protect against bile leak ( Video 1 ). The patient was observed overnight and discharged without complications.

This case highlights the utility of the biliary rendezvous technique for deep biliary access in case of an invisible ampulla, the need for a dual-channel catheter for deep cannulation of the bile duct in rendezvous procedures, and the value of primary sphincteroplasty for stone extraction in cases where sphincterotomy is deemed unsafe or impossible.

\section{Video 1}

Demonstration of the endoscopic ultrasound (EUS)-guided biliary rendezvous procedure with biliary sphincteroplasty, stone extraction, and stent insertion.

\section{Endoscopy_UCTN_Code_TTT_1AS_2AD}

Competing interests: None

\section{Jayapal Ramesh, Juan P. Gutiérrez, Charles M. Wilcox}

Division of Gastroenterology and Hepatology, University of Alabama at Birmingham, Birmingham, Alabama, United States of America

\section{Reference}

1 Shah JN, Marson F, Weilert $F$ et al. Single-operator, single-session EUS-guided anterograde cholangiopancreatography in failed ERCP or inaccessible papilla. Gastrointest Endosc 2012; 75: 56-64

Bibliography

DOI http://dx.doi.org/

$10.1055 / \mathrm{s}-0033-1344833$

Endoscopy 2013; 45: E349

(c) Georg Thieme Verlag KG

Stuttgart · New York

ISSN 0013-726X

\section{Corresponding author \\ Jayapal Ramesh}

Basil Hirschowitz Endoscopic Center of Excellence Division of Gastroenterology-Hepatology University of Alabama at Birmingham BDB 389

1808 7th Avenue South

Birmingham

Alabama 35294

USA

Fax: +1-205-9756381

j1ramesh@gmail.com 